\title{
A PROBLEM ON VISCOUS FLUID MOTION BETWEEN TWO FIXED CYLINDERS
}

\author{
S. K. SEN \\ Research Center for Mathematical and Physical Sciences (RCMPS) \\ University of Chittagong, Chittagong-4331, Bangladesh. \\ $A N D$ \\ M. J. AHAMMAD \\ Departmnet of Mathematics, University of Chittagong, Chittagong-4331, Bangladesh.
}

\begin{abstract}
A problem on the two dimensional slow viscous fluid motion obeying the Stokes equations is solved in terms of the Earnshaw stream function, when a line source and equal line sink are arbitrarily situated in a viscous fluid bed between two fixed co-axial circular cylinders. Fluid mechanical properties of interest, such as drags and torques acting upon the cylinders are calculated. Also we have shown the variation of the forces per unit length on the inner cylinder with its radius keeping outer cylinder fixed, whose radius is assumed to be one.
\end{abstract}

\section{INTRODUCTION}

Ranger [1] has solved the problem of a slow viscous flow between two fixed co-axial circular cylinders, due to a line source and equal line sink located at arbitrary points on the outer boundary with the help of the Earnshaw stream function; this is in fact an extension of Rayleigh [2] problem which consists of the two-dimensional Stokes flow within a circular cylinder due to a line source and sink of equal strength situated at opposite ends of a diameter. The two-dimensional Stokes flow inside and outside a circular cylinder induced by a pair of line singularities (rotlets or Stokeslets) has been extensively studied by Daripa and Palaniappan [3]. Relevantly, Dorrepaal et al [4] showed that a uniform stream emerges when a rotlet or a Stokeslet is located in front of a circular cylinder in a viscous fluid. Similarly, Smith [5] and Avudainayagam et al [6] also found that, when the potential flow singularity such as a line source or a sink etc., is placed before a fixed circular cylinder, a uniform stream is generated at large distances from the cylinder. Chowdhury and Sen [7] have analytically solved the problem of Stokes flow due to the presence of a rotlets or Stokeslets inside a circular cylinder by taking the partial support of the circle theorem for external Stokes flow in Sen [8]. Ranger [9] extensively studied the flow structure within a circular cylinder due to a line rotlet. Again for the three-dimensional analogous cases (i.e. viscous fluid motions within a spherical container) some literature is available; in this respect Collins $[10,11]$ for axi-symmetrical viscous fluid motion, and Palaniappan et al [12] for the non-axi-symmetrical fluid are remarkable. Hacborn et al [13] have solved the problem of a three-dimensional rotlets in viscous fluid within a sphere, highlighting the phenomenon of separation and flow reversal. Later Shail [14] rederived the solution of the same problem by a relatively easy method, and also gave the solution of the problem of the Stokeslets within a spherical container. Here it is noteworthy that when the singularities are placed in viscous fluid between two cylinders or spherical boundaries or different ones, the solutions of the corresponding flow problems are not available in the literature. In an attempt towards solving such problems, we first present in this paper the solution to the problem of the 
viscous fluid motion due to the presence of a line source and a sink of equal strength between two co-axial circular cylinders.

\section{SLOW VISCOUS FLOW WITHIN A CIRCULAR CYLINDER}

At first it is necessary to determine the flow due to the combination of a source of strength $m$ at the point $\mathrm{A}\left(a_{1}, \alpha\right)$ and a sink of the same strength at the point $\mathrm{B}\left(a_{1}\right.$, $\alpha$ ), where $a_{1}<a, a$ being the radius of the circular cylinder.

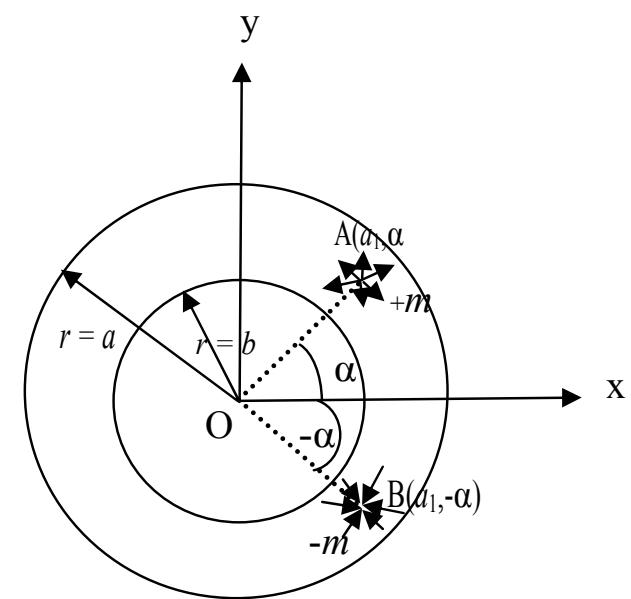

Fig. 1. Sketch of the flow along the fluid bed between two co-axial circular cylinders.

Now the basic stream function due to these singularities in the absence of rigid boundaries is given by Thomson [15]

$\psi_{0}(r, \theta)=-m\left\{\tan ^{-1}\left(\frac{r \sin \theta-a_{1} \sin \alpha}{r \cos \theta-a_{1} \cos \alpha}\right)-\tan ^{-1}\left(\frac{r \sin \theta+a_{1} \sin \alpha}{r \cos \theta-a_{1} \cos \alpha}\right)\right\}$.

When the stream function $\psi_{0}(\mathrm{r}, \theta)$ is expanded for large $r$, we find that

$\psi_{0}(r, \theta)=-m\left\{\theta-\sum_{n=1}^{\infty} \frac{1}{n}\left(\frac{a_{1}}{r}\right)^{n} \sin n(\alpha-\theta)\right\}+m\left\{\theta-\sum_{n=1}^{\infty} \frac{1}{n}\left(\frac{a_{1}}{r}\right)^{n} \sin n(\alpha+\theta)\right\}$.

Here we observe that $\psi_{0}(r, \theta) \sim O\left(\frac{1}{r}\right)$ for large $r$. Thus the circle theorem I for the interior Stokes flow in Sen [8] and Golam [16] applies. If the cylinder $r=a$ is introduced the flow field of the singularities, the stream function for the flow interior to the cylinder is given by the formula 
$\psi_{1}(r, \theta)=\psi_{0}(r, \theta)-\psi_{0}\left(\frac{a^{2}}{r}, \theta\right)+\frac{\left(r^{2}-a^{2}\right)}{a^{2}} \frac{\partial}{\partial r} \psi_{0}\left(\frac{a^{2}}{r}, \theta\right)$

Thus substituting the basic stream (2.1) in (2.3) yields the stream function, which is given by

$$
\begin{aligned}
& \psi_{1}(r, \theta)=-m\left\{\tan ^{-1}\left(\frac{r \sin \theta-a_{1} \sin \alpha}{r \cos \theta-a_{1} \cos \alpha}\right)-\tan ^{-1}\left(\frac{r \sin \theta+a_{1} \sin \alpha}{r \cos \theta-a_{1} \cos \alpha}\right)\right\} \\
& -m\left\{\tan ^{-1}\left(\frac{r \sin \theta-\frac{a^{2}}{a_{1}} \sin \alpha}{r \cos \theta-\frac{a^{2}}{a_{1}} \cos \alpha}\right)-\tan ^{-1}\left(\frac{r \sin \theta+\frac{a^{2}}{a_{1}} \sin \alpha}{r \cos \theta-\frac{a^{2}}{a_{1}} \cos \alpha}\right)\right\}-m \frac{\left(r^{2}-a^{2}\right) r}{a_{1}}\left\{\frac{\sin (\theta-\alpha)}{R_{1}^{2}}-\frac{\sin (\theta+\alpha)}{R_{2}^{2}}\right\}^{,}
\end{aligned}
$$

where $R_{1}=\left\{r^{2}+\frac{a^{4}}{a^{2}{ }_{1}}-2 r \frac{a^{2}}{a_{1}} \cos (\theta-\alpha)\right\}^{1 / 2}$ is the distance between the points $(r, \theta)$ and $\left(\frac{a^{2}}{a_{1}}, \alpha\right)$, and $R_{2}=\left\{r^{2}+\frac{a^{4}}{a_{1}^{2}}-2 r \frac{a^{2}}{a_{1}} \cos (\theta+\alpha)\right\}^{1 / 2}$ is the distance between the points $(r, \theta)$ and $\left(\frac{a^{2}}{a_{1}},-\alpha\right)$.

Now to use the stream function i.e. $\psi_{1}(r, \theta)$ in next section we present it in a summation form as

$\psi_{1}=\sum_{n=1}^{\infty} f_{n}(r) \cos n \theta$,

where

$f_{n}(r)=2 m\left[-\frac{1}{n}\left(\frac{1}{a_{1}}\right)^{n}+\left(\frac{a_{1}}{a^{2}}\right)^{n}\left\{\frac{1}{n}-\left(1-\frac{r^{2}}{a^{2}}\right)\right\}\right] r^{n} \sin n \alpha$.

\section{THE FLOW BETWEEN A PAIR OF CO-AXIAL CYLINDERS}

Now we may introduce a cross-section of a circular cylinder with radius $b(<a)$ into the flow and is fixed in a co-axial position with respect to the outer cylinder. As a result of this situation, the Earnshaw stream function for a new flow field in the region common to the cylinders becomes, say

$$
\psi=\psi_{1}+\psi_{2},
$$

where $\psi_{2}$ is a suitable general solution of the equation

$$
\nabla_{1}^{4} \psi_{2}=0 .
$$

given by $\psi_{2}(r, \theta)=g_{1}(r) \cos \theta+\sum_{n=2}^{\infty} g_{n}(r) \cos n \theta$, 
where $\quad g_{1}(r)=A_{1} r+B_{1} r^{3}+\frac{C_{1}}{r}+D_{1} r \log r$,

and $\quad g_{n}(r)=A_{n} r^{n}+B_{n} r^{-n}+C_{n} r^{n+2}+D_{n} r^{-n+2}, n>1$.

$A_{1}, B_{1}, C_{1}, D_{1}, A_{n}, B_{n}, C_{n}$, and $D_{n}$ being constants to be determined.

The stream function $\psi_{1}$ satisfies the condition of the flow interior to the outer boundary ; $r=a$.

Hence it is sufficient that the stream function $\psi_{2}$ must also satisfy the following conditions

on $r=a, \psi_{2}=\frac{\partial \psi_{2}}{\partial r}=0$,

Again, on the inner boundary, the stream function $\psi$, represented by equation (3.1) must satisfy the following boundary conditions,

on $r=b, \psi=\frac{\partial \psi}{\partial r}=0$,

Now using the boundary conditions (3.6), we have two cases,

one case $\psi_{2}=0$ on $r=a$, which implies

$g_{1}(a)=0, g_{n}(a)=0, n=2,3,4, \ldots$

And for another case, $\frac{\partial \psi_{2}}{\partial r}=0$ on $r=a$,

which implies

$$
g_{1}^{\prime}(a)=0, g_{n}^{\prime}(a)=0,
$$

Now in order to use the boundary conditions (3.7), we express the stream function (3.1) in the following form, incorporating the stream function (2.6) and (3.3).

$\psi(r, \theta)=\left\{f_{1}(r)+g_{1}(r)\right\} \cos \theta+\sum_{n=2}^{\infty}\left\{f_{n}(r)+g_{n}(r)\right\} \cos n \theta$

Now the boundary condition $\psi(r, \theta)=0$ on $r=b$, yields

$f_{1}(b)+g_{1}(b)=0, f_{n}(b)+g_{n}(b)=0$.

Again, the boundary condition $\frac{\partial \psi}{\partial r}=0$ on $r=b$, gives

$f_{1}^{\prime}(b)+g_{1}^{\prime}(b)=0, f_{n}^{\prime}(b)+g_{n}^{\prime}(b)=0$,

where the prime denotes differentiation with respect to $r$.

Now equations (3.8), (3.9), (3.11) and (3.12) constitute a set of following linear equations to determine the coefficients $A_{1}, B_{1}, C_{1}$ and $D_{1}$.

$$
\begin{aligned}
& A_{1} a+B_{1} a^{3}+\frac{C_{1}}{a}+D_{1} a \log a=0, \\
& A_{1} a+3 B_{1} a^{3}-\frac{C_{1}}{a}+D_{1} a(\log a+1)=0, \\
& A_{1} b+B_{1} b^{3}+\frac{C_{1}}{b}+D_{1} b \log b=-f_{1}(b),
\end{aligned}
$$




$$
A_{1} b+3 B_{1} b^{3}-\frac{C_{1}}{b}+D_{1} b(\log b+1)=-b f_{1}^{\prime}(b) .
$$

Also equations (3.8), (3.9), (3.11) and (3.12) constitute another set of following linear equations to determine the coefficients $A_{n}, B_{n}, C_{n}$ and $D_{n}$ where $n=2,3,4, \ldots$ etc. Thus we have

$$
\begin{aligned}
& A_{n} a^{n}+\frac{B_{n}}{a^{n}}+C_{n} a^{n+2}+\frac{D_{n}}{a^{n-2}}=0, \\
& n A_{n} a^{n}-\frac{n B_{n}}{a^{n}}+(n+2) C_{n} a^{n+2}-\frac{(n-2) D_{n}}{a^{n-2}}=0, \\
& A_{n} b^{n}+\frac{B_{n}}{b^{n}}+C_{n} b^{n+2}+\frac{D_{n}}{b^{n-2}}=-f_{n}(b), \\
& n A_{n} b^{n}-\frac{n B_{n}}{b^{n}}+(n+2) C_{n} b^{n+2}-\frac{(n-2) D_{n}}{b^{n-2}}=-b f_{n}^{\prime}(b) .
\end{aligned}
$$

On solving the equations (3.13), (3.14), (3.15) and (3.16), we obtain the constants as shown below.

$$
\begin{aligned}
& A_{1} l_{1}=\frac{m \sin \alpha}{a_{1} a^{2}}\left\{(1-2 \log a)\left(a^{4}-2 a_{1}^{2} b^{2}\right)-a^{2} b^{2}(1+2 \log a)-4 a_{1}^{2} b^{4}\left(a^{2}-b^{2}\right)^{-1} \log \frac{b}{a}\right\}, \\
& B_{1} l_{1}=\frac{m \sin \alpha}{a_{1} a^{4}\left(a^{2}-b^{2}\right)}\left\{\left(2 a_{1}^{2} b^{2}-a^{4}\right)\left(a^{2}-b^{2}\right)+2 a_{1}^{2} b^{4} \log \frac{b}{a}\right\}, \\
& C_{1} l_{1}=\frac{m b^{2} \sin \alpha}{a_{1}\left(a^{2}-b^{2}\right)}\left\{a^{2}\left(a^{2}-b^{2}\right)+2 a_{1}^{2} b^{2} \log \frac{b}{a}\right\}, \\
& D_{1} l_{1}=\frac{-2 m \sin \alpha}{a_{1} a^{2}}\left\{2 a_{1}^{2} b^{2}-a^{2}\left(a^{2}+b^{2}\right)\right\}, \\
& \text { where } l_{1}=\left\{\left(a^{2}+b^{2}\right) \log \frac{b}{a}+\left(a^{2}-b^{2}\right)\right\} .
\end{aligned}
$$

Also solving the equations (3.17), (3.18), (3.19) and (3.20) yields,

$$
\begin{aligned}
& A_{n} l_{n}=\left[a ^ { 2 n } b ^ { n - 2 } ( n + 1 ) \{ a ^ { 2 n + 2 } ( a ^ { - 2 n } - b ^ { - 2 n } ) + n ( a ^ { 2 } - b ^ { 2 } ) \} ^ { - 1 } \left[\frac { 1 } { 2 n } \left\{a^{2} b^{2 n}\left(a^{-2 n}-b^{-2 n}\right)\right.\right.\right. \\
& \left.\left.\left.+n\left(a^{2}-b^{2}\right)\right\} n^{2} b^{2}+2 n b^{2}-n^{2} a^{2}-n a^{2}-n a^{2 n+2} b^{-2 n}\right\}-\left\{a^{-2 n+2} b^{-2 n+2}\left(a^{2 n}-b^{2 n}\right)^{2}-n^{2}\left(a^{2}-b^{2}\right)^{2}\right\}\right] \\
& \left.-\frac{1}{2 n} b^{3 n-2}\left\{n^{2} b^{2}+2 n b^{2}-n^{2} a^{2}-n a^{2}-n a^{2 n+2} b^{-2 n}\right\}\right] f_{n}(b) \\
& +\frac{1}{2 n} b^{n-1}\left(n b^{2}-n a^{2}+a^{2 n+2} b^{-2 n}-a^{2}\right)\left[( n - 1 ) \left[\left\{a^{2 n+2}\left(a^{-2 n}-b^{-2 n}\right)+n\left(a^{2}-b^{2}\right)\right\}^{-1}\right.\right. \\
& \left.\left.\left\{a^{2 n+2} b^{2 n}\left(a^{-2 n}-b^{-2 n}\right)+n a^{2 n}\left(a^{2}-b^{2}\right)\right\}+b^{2 n}\right]+n b^{2 n}\right] f_{n}^{\prime}(b), \\
& B_{n} l_{n}=\frac{1}{2 n} a^{2 n} b^{n-2}\left[\left[n a ^ { 2 n } \{ a ^ { 2 n + 2 } ( a ^ { - 2 n } - b ^ { - 2 n } ) + n ( a ^ { 2 } - b ^ { 2 } ) \} ^ { - 1 } \left[2 \left\{a^{-2 n+2} b^{-2 n+2}\left(a^{2 n}-b^{2 n}\right)^{2}\right.\right.\right.\right.
\end{aligned}
$$




$$
\begin{aligned}
& \left.-n^{2}\left(a^{2}-b^{2}\right)^{2}\right\}-\left\{a^{2} b^{2 n}\left(a^{-2 n}-b^{-2 n}\right)+n\left(a^{2}-b^{2}\right)\right\}\left\{n^{2} b^{2}+2 n b^{2}-n^{2} a^{2}\right. \\
& \left.\left.\left.-n a^{2}-n a^{2 n+2} b^{-2 n}\right\}\right]-b^{2 n}(n-1)\left(n^{2} b^{2}+2 n b^{2}-n^{2} a^{2}-n a^{2}-n a^{2 n+2} b^{-2 n}\right)\right] f_{n}(b) \\
& \left.+\left(n b^{2}-n a^{2}+a^{2 n+2} b^{-2 n}-a^{2}\right)\left[n a^{2 n} b\left\{a^{2 n+2}\left(a^{-2 n}-b^{-2 n}\right)+n\left(a^{2}-b^{2}\right)\right\}^{-1}+(n-1) b^{2 n+1}\right] f_{n}^{\prime}(b)\right], \quad(3.27) \\
& C_{n} l_{n}=\frac{-1}{2} a^{2 n-2} b^{n-2}\left\{a^{2 n+2}\left(a^{-2 n}-b^{-2 n}\right)+n\left(a^{2}-b^{2}\right)\right\}^{-1}\left[\left\{a^{2} b^{2 n}\left(a^{-2 n}-b^{-2 n}\right)\right.\right. \\
& \left.+n\left(a^{2}-b^{2}\right)\right\}\left\{\left(n^{2} b^{2}+2 n b^{2}-n^{2} a^{2}-n a^{2}-n a^{2 n+2} b^{-2 n}\right) f_{n}(b)-b\left(n b^{2}-n a^{2}+a^{2 n+2} b^{-2 n}-a^{2}\right) f_{n}^{\prime}(b)\right\} \\
& \left.-2 n\left\{a^{-2 n+2} b^{-2 n+2}\left(a^{2 n}-b^{2 n}\right)^{2}-n^{2}\left(a^{2}-b^{2}\right)^{2}\right\} f_{n}(b)\right],
\end{aligned}
$$

and

$D_{n} l_{n}=\frac{1}{2} a^{2 n-2} b^{3 n-2}\left[\left(n^{2} b^{2}+2 n b^{2}-n^{2} a^{2}-n a^{2}-n a^{2 n+2} b^{-2 n}\right) f_{n}(b)-b\left(n b^{2}-n a^{2}+a^{2 n+2} b^{-2 n}-a^{2}\right) f_{n}^{\prime}(b)\right]$,

where $l_{n}=\left\{\left(a^{2 n}-b^{2 n}\right)^{2}-n^{2} a^{2 n-2} b^{2 n-2}\left(a^{2}-b^{2}\right)^{2}\right\}$.

Now the evaluated stream function for the flow pattern in the region bounded by the concentric cylinders takes the form

$$
\psi=\psi_{1}+\psi_{2}
$$

where

$$
\begin{aligned}
& \psi_{1}(r, \theta)=-m\left\{\tan ^{-1}\left(\frac{r \sin \theta-a_{1} \sin \alpha}{r \cos \theta-a_{1} \cos \alpha}\right)-\tan ^{-1}\left(\frac{r \sin \theta+a_{1} \sin \alpha}{r \cos \theta-a_{1} \cos \alpha}\right)\right\} \\
& -m\left\{\tan ^{-1}\left(\frac{r \sin \theta-\frac{a^{2}}{a_{1}} \sin \alpha}{r \cos \theta-\frac{a^{2}}{a_{1}} \cos \alpha}\right)-\tan ^{-1}\left(\frac{r \sin \theta+\frac{a^{2}}{a_{1}} \sin \alpha}{r \cos \theta-\frac{a^{2}}{a_{1}} \cos \alpha}\right)\right\}-m \frac{\left(r^{2}-a^{2}\right) r}{a_{1}}\left\{\frac{\sin (\theta-\alpha)}{R_{1}^{2}}-\frac{\sin (\theta+\alpha)}{R^{2}{ }_{2}}\right\}
\end{aligned}
$$

and

$\psi_{2}=\left\{A_{1} r+B_{1} r^{3}+\frac{C_{1}}{r}+D_{1} r \log r\right\} \cos \theta+\sum_{n=2}^{\infty}\left\{A_{n} r^{n}+B_{n} r^{-n}+C_{n} r^{n+2}+D_{n} r^{-n+2}\right\} \cos n \theta$,

the values of the constants $A_{1}, B_{1}, C_{1} \& D_{1}$ and $A_{n}, B_{n}, C_{n} \& D_{n}$ being given in (3.21), (3.22), (3.23) and (3.24) also (3.26), (3.27), (3.28) and (3.29).

\section{DRAGS AND TORQUES ON THE BOTH CYLINDERS}

The components of the forces exerted on the inner and outer cylinders can be expressed as $\left(X_{1}, Y_{1}\right),\left(X_{2}, Y_{2}\right)$ respectively, and that for the torque is $\left(T_{1}, T_{2}\right)$ which can be calculated with the help of Ranger [1] and these are

$$
X_{1}=\mu b^{2} \int_{0}^{2 \pi}\left(\frac{\partial^{3} \psi}{\partial r^{3}}\right)_{r=b} \sin \theta d \theta, \quad X_{2}=\mu a^{2} \int_{0}^{2 \pi}\left(\frac{\partial^{3} \psi}{\partial r^{3}}\right)_{r=a} \sin \theta d \theta
$$


$Y_{1}=-\mu b^{2} \int_{0}^{2 \pi}\left(\frac{\partial^{3} \psi}{\partial r^{3}}\right)_{r=b} \cos \theta d \theta, \quad Y_{2}=-\mu a^{2} \int_{0}^{2 \pi}\left(\frac{\partial^{3} \psi}{\partial r^{3}}\right)_{r=a} \cos \theta d \theta$

$T_{1}=\mu b^{2} \int_{0}^{2 \pi}\left(\frac{\partial^{2} \psi}{\partial r^{2}}\right)_{r=b} d \theta^{\prime} T_{2}=\mu a^{2} \int_{0}^{2 \pi}\left(\frac{\partial^{2} \psi}{\partial r^{2}}\right)_{r=a} d \theta^{\prime}$

where $\psi=\left\{f_{1}(r)+g_{1}(r)\right\} \cos \theta+\sum_{n=2}^{\infty}\left\{f_{n}(r)+g_{n}(r)\right\} \cos n \theta$.

Here we see that the force components $X_{1}$ and $X_{2}$ represented by equations (4.1), which will be zero for all positive integers.

$$
X_{1}=X_{2}=0 \text {. }
$$

And, then other force components represented by (4.2), will appear, on the evaluation as

$$
Y_{1}=-\pi \mu b^{2}\left\{f_{1}^{\prime \prime \prime}(b)+g_{1}^{\prime \prime \prime}(b)\right\},
$$

and $Y_{2}=-\pi \mu a^{2}\left\{f_{1}^{\prime \prime \prime}(a)+g_{1}^{\prime \prime \prime}(a)\right\}$,

Finally, with the assistance of the equations (2.7) and (3.4) the force components (4.6) appears as,

$Y_{1}=\frac{-4 m \pi \mu b^{2} \sin \alpha}{a_{1} a^{4}}\left[3 a_{1}^{2}+\frac{1}{l_{1}}\left\{a_{1}^{2}\left(a^{2}+3 b^{2}\right)-\left(a^{2}+b^{2}\right)\left(2 a^{4} b^{-2}+3 a_{1}^{2} \log \frac{b}{a}\right)\right]\right.$,

and $Y_{2}=\frac{-4 m \pi \mu \sin \alpha}{a_{1} a^{2}}\left[3 a_{1}^{2}+\frac{2}{l_{1}}\left\{2 a_{1}^{2} b^{2}-a^{2}\left(a^{2}+b^{2}\right)\right\}\right]$.

After calculation the torques equation (4.5) which appear as zero,

i.e., $T_{1}=T_{2}=0$.

Now by taking the locations of the singularities at the points ${ }_{A}\left(\frac{3}{4}, \alpha\right)$ and $B\left(\frac{3}{4},-\alpha\right)$, we now display the variation of the forces $Y_{1}$ and $Y_{2}$ with the change of the inner boundary, keeping the radius of the outer boundary fixed with constant radius unity, i.e. $a_{1}=\frac{3}{4}$, $a=1$, then the equation (4.7) and (4.8) become,

$Y_{1}^{(i)}=\frac{-Y_{1}}{4 m \mu \pi \sin \alpha}=\frac{4 b^{2}}{3}\left[\frac{27}{16}+\frac{1}{l_{1}^{(i)}}\left\{\frac{9}{16}\left(1+3 b^{2}\right)-\left(1+b^{2}\right)\left(2 b^{-2}+\frac{27}{16} \log b\right)\right\}\right]$,

and $Y_{2}^{(i)}=\frac{-Y_{2}}{4 m \pi \mu \sin \alpha}=\frac{4}{3}\left[\frac{27}{16}+\frac{1}{l_{1}^{(i)}}\left\{\frac{9 b^{2}}{8}-\left(1+b^{2}\right)\right\}\right]$,

where $l_{1}^{(i)}=\left\{\left(1+b^{2}\right) \log b+\left(1-b^{2}\right)\right\}$.

Some numerical results are displayed in the Table 2.1 and Table 2.2 below. 


\begin{tabular}{|c|c|}
\hline \multicolumn{2}{|c|}{$\begin{array}{l}\text { Table 2.1. Numerical values of the } \\
\text { force } Y_{1}^{(i)} \text { on the inner variable circular } \\
\text { boundary when its radius changes with in } \\
\text { the domain } 0<b<3 / 4\end{array}$} \\
\hline $\mathrm{b}$ & $Y_{1}^{(i)}$ \\
\hline 0 & 0 \\
\hline 0.1 & 1.994093673 \\
\hline 0.2 & 3.717114017 \\
\hline 0.3 & 6.553486701 \\
\hline 0.4 & 11.724385770 \\
\hline 0.5 & 22.187109330 \\
\hline 0.6 & 46.5375322502 \\
\hline 0.7 & 116.729102000 \\
\hline
\end{tabular}

\begin{tabular}{|c|c|}
\hline \multicolumn{2}{|c|}{ Table 2.2. Numerical values of the force } \\
$Y_{2}^{(i)}$ on the outer boundary of fixed \\
radius unity with the variation of the inner \\
boundary. \\
\hline $\mathrm{b}$ & $Y_{2}^{(i)}$ \\
\hline 0 & 0 \\
\hline 0.1 & 3.246375430 \\
\hline 0.2 & 4.108557009 \\
\hline 0.3 & 5.526743387 \\
\hline 0.4 & 8.112192883 \\
\hline 0.5 & 13.343554670 \\
\hline 0.6 & 25.518769440 \\
\hline 0.7 & 60.614559800 \\
\hline
\end{tabular}

Finally, it is of interest to study the variation of the forces $Y_{1}$ and $Y_{2}$ with the change of the outer boundary, keeping the radius of the inner boundary fixed with constant radius unity, i.e., $a_{1}=\frac{3}{4}, b=1$, then the equation (4.24) and (4.25) yields,

$$
\begin{aligned}
& Y_{1}^{(i i)}=\frac{-Y_{1}}{4 \pi m \mu \sin \alpha}=\frac{4}{3 a^{4}}\left[\frac{27}{16}+\frac{1}{l_{1}^{(i)}}\left\{\frac{9}{16}\left(a^{2}+3\right)-\left(1+a^{2}\right)\left(2 a^{4}-\frac{27}{16} \log a\right)\right\}\right] \\
& \text { and } Y_{2}^{(i i)}=\frac{-Y_{2}}{4 \pi m \mu \sin \alpha}=\frac{4}{3 a^{2}}\left[\frac{27}{16}+\frac{2}{l_{1}^{(i i)}}\left\{\frac{9}{8}-a^{2}\left(1+a^{2}\right)\right\}\right]
\end{aligned}
$$

where $l_{1}^{(i i)}=\left\{\left(a^{2}-1\right)-\left(a^{2}+1\right) \log a\right\}$.

Again some numerical results are exhibited in the Table 2.3 and Table 2.4 below.

Table 2.3. Numerical values of the force $Y_{1}$ on the outer variable circular boundary when its radius changes with in the domain, $1.2 \leq a<\infty$.

\begin{tabular}{|c|c|}
\hline $\mathrm{a}$ & $Y_{1}^{(i i)}$ \\
\hline 1.2 & 909.2905125 \\
\hline 1.4 & 176.9495784 \\
\hline 1.6 & 75.50272684 \\
\hline 1.8 & 41.1582351 \\
\hline 2 & 27.01817335 \\
\hline 3 & 8.818570195 \\
\hline 4 & 5.26973404 \\
\hline 5 & 3.87800259 \\
\hline 6 & 3.150120352 \\
\hline 7 & 2.703534576 \\
\hline 8 & 2.401296744 \\
\hline 9 & 2.182533275 \\
\hline 10 & 2.014973052 \\
\hline 100 & 0.739637405 \\
\hline 1000 & 0.451383924 \\
\hline
\end{tabular}

Table 2.4. Numerical values of the force $Y_{2}$ on the outer variable circular boundary when its radius changes with in the domain, $1.2 \leq a<\infty$.

\begin{tabular}{|c|c|}
\hline $\mathrm{a}$ & $Y_{2}^{(i i)}$ \\
\hline 1.2 & 910.8531204 \\
\hline 1.4 & 178.0975376 \\
\hline 1.6 & 74.3816331 \\
\hline 1.8 & 41.8526700 \\
\hline 2 & 27.5806734 \\
\hline 3 & 9.0685700 \\
\hline 4 & 5.4103590 \\
\hline 5 & 3.9685003 \\
\hline 6 & 3.2126204 \\
\hline 7 & 2.7494529 \\
\hline 8 & 2.4364530 \\
\hline 9 & 2.210311 \\
\hline 10 & 2.0388307 \\
\hline 100 & 0.7398624 \\
\hline 1000 & 0.4513562 \\
\hline
\end{tabular}




\section{DISCUSSION OF THE NUMERICAL RESULTS}

In the both sets of the tables, we observe that the force on the both cylinders is gradually increasing when the cylinders become closure to each other and decreasing in the reverse case, as was to be expected.

\section{REFERENCES}

1. K. B. Ranger, A problem on the slow motion of a viscous fluid between two fixed cylinders, Q. J Appl. Math. Vol. XIV, pt 4, (1961), 411-421.

2. Lord Rayleigh, Scientific papers (vol. iv). (1893), p.78.

3. P. Darip and D. Palaniappan, Singularity induced exterior and interior Stokes flows, Phys. Fluids, vol.13 (ii), (2001), 3134-3154.

4. J. M. Dorrepaal , M. E. O' Neill and K. B. Ranger, Two dimensional stokes flows with cylinders and line singularities, Mathematika , 31 (1984), 65-75.

5. S. H. Smith, Some Limitation of two dimensional unbounded stokes flow, Phys. Fluid, A2 (1990), 1724-1730.

6. A. Avudaonayagam and B. Jothiram, No - Slip images of certain line singularities in a circular cylinder, Int. J. Eng. Sci., 25 (1987), 1193 - 1205.

7. G. A. H. Chowdhury and S. K. Sen , A note on Stokes flow within a circular cylinder, GANIT , J. Bangladesh Math. Soc., 26 (2006), 43-50.

8 S. K. Sen , Circle Theorems for steady Stokes flow , Z. Angew Math. Phys. (ZAMP) 40, (1989), 139-146.

9. K. B. Ranger, Eddies in two - dimensional stokes flow, Int. J. Eng. Sci., 18 (1980) 181-190.

10. W.D. Collins, A note on Stokes stream function for the slow steady motion of viscous fluid before plane and spherical boundaries, Mathematika, 1 (1954), 125-130.

11. W.D.Collins, Note on a sphere theorem for axi-symmetrical stokes flow of viscous fluid, Mathematika, 5 (1958), 118-121.

12. D. Palaniappan, S.D. Nigam and T. Amaranath, Lamb's solution of Stokes's equations: A sphere theorem, Q. JL Mech. Appl. Math., 45, pt .1 (1992), 47-56.

13. W. Hacborn, M.E. O'neill and K.B. Ranger, The structure of an asymmetric stokes flow , Q. J. Mech. appl. Math., 39,pt.1 (1986),1-14.

14. R Shail, A note on some asymmetric stokes flow with in a sphere ; Q.J. Mech. appl. Math. , 40, pt. 2,(1987), 223-233.

15. L. M. Milne Thomson, Theoretical Hydrodynamics, 5th edition, MacMillan Ltd. London, 1972.

16. G.A.H. Chowdhury, PhD Thesis, 2001, RCMPS,University of Chittagong, Chittagong. Bangladesh.

Journal of Bangladesh Academy of Sciences, Vol. 33, No. 1, 107-115, 2009 